\title{
Stabilization of solutions to a differential-delay equation in a Banach space
}

\author{
by J. J. Koliha (Melbourne, Vic.) and Ivan StrašKraba (Praha)
}

\begin{abstract}
A parameter dependent nonlinear differential-delay equation in a Banach space is investigated. It is shown that if at the critical value of the parameter the problem satisfies a condition of linearized stability then the problem exhibits a stability which is uniform with respect to the whole range of the parameter values. The general theorem is applied to a diffusion system with applications in biology.
\end{abstract}

1. Introduction. In this work we investigate a class of parameter dependent differential-delay equations in a Banach space $X$ and apply the method of fixed points in the spaces of functions in $X$ tending to zero as $t \rightarrow \infty$ at an appropriate rate that was developed in [3]. In particular, we address the stability of the stationary solution of such an equation. The stability is shown to be uniform with respect to a small parameter on some finite interval.

The stability of solutions to differential-delay equations has been studied in a number of publications. Let us mention at least a few of them. The asymptotic stability for Problem (2.1) below with $\varepsilon=1$ has been proved in $[6,7]$ under the assumption of the stability of the linearized problem. The results are applied to a parabolic equation with delay. In [10] stabilization of solutions to the fully nonlinear problem is established by means of monotonicity of the generator of the corresponding nonlinear semigroup. A similar approach is also used in [2], where a series of results on asymptotic behavior of solutions and their mean values is proved. Finally, in $[8,9]$ appropriate functionals and sufficiently strong a priori bounds are used to show the (uniform) asymptotic stability of solutions under certain natural assumptions.

1991 Mathematics Subject Classification: Primary 34D15, 34G20; Secondary 47H15.

Key words and phrases: abstract differential-delay equation, dependence on parameter, uniform stability.

Supported by the Australian Research Council Grant S6969557. The second author was partially supported by the Czech Republic Grant Agency Grant 201/93/2177. 
In our approach the existence and stabilization of solution is shown by a fixed point argument. We obtain the rate of convergence and describe the global behavior of solution in connection with a singular parameter involved.

Our notation is consistent with that introduced in [3]; in particular, we adopt the usual notation $L^{p}(M ; X)$ for the $L^{p}$-spaces of functions from a set $M \subset \mathbb{R}^{N}$ into a Banach space $X, W^{k, p}(M ; X)$ for the Sobolev spaces of $k$ th order, $C^{k}(M ; X)$ for the spaces of functions with continuous derivatives up to order $k, L(X, Y)$ for the space of the continuous linear operators from $X$ into $Y$ with $L(X)=L(X, X), L_{\mathrm{s}}(X)$ being $L(X)$ equipped with the strong operator topology, and so on.

2. Formulation of the problem. Let us consider the following parameter dependent problem:

$$
\begin{gathered}
\varepsilon u_{\varepsilon}^{\prime}(t)+A u_{\varepsilon}(t)-E u_{\varepsilon}(t-\tau)=F u_{\varepsilon}(t)+G u_{\varepsilon}(t-\tau), \quad t>0 \\
u_{\varepsilon}(s)=x(s), \quad s \in(-\tau, 0], \varepsilon \in\left[0, \varepsilon_{0}\right]\left(\tau>0, \varepsilon_{0}>0\right),
\end{gathered}
$$

where $A: X \supset D(A) \rightarrow X$ is linear, $E \in L(X), F, G: X \rightarrow X$ are possibly nonlinear operators. The fixed number $\tau>0$ is a given delay, $\varepsilon \in\left[0, \varepsilon_{0}\right]$ a parameter, and $x(\cdot):(-\tau, 0] \rightarrow X$ a given initial datum. We are interested in the stabilization of $u_{\varepsilon}(t)$ as $t \rightarrow \infty$. This will be achieved by an appropriate splitting of the problem in a stable linear part and a nonlinear perturbation which is locally small. We shall work in the space

$$
\begin{aligned}
& L_{w}^{\infty}(0, \infty ; X) \\
& \quad=\left\{u \in L^{\infty}(0, \infty ; X):\|u\|_{w}:=\underset{t \geq 0}{\operatorname{essup} \sup } w(t)|u(t)|<\infty\right\},
\end{aligned}
$$

for some function $w \in L_{\mathrm{loc}}^{\infty}(0, \infty)$ such that $w(t) \geq 1$ a.e. in $(0, \infty)$ and $\lim _{t \rightarrow \infty} w(t)=\infty$. It is a standard result that the space $L_{w}^{\infty}(0, \infty ; X)$ is a Banach space under the norm $\|\cdot\|_{w}$.

We make the following assumptions:

$$
\left\{\begin{array}{l}
\text { (i) }-A \text { is the generator of a } C_{0} \text {-semigroup in } L(X) ; \\
\text { (ii) } F: X \rightarrow X, F(0)=0 ; \\
\text { (iii) the semigroup } T(t) \text { generated by }-A \text { satisfies } \\
|T(t)| \leq \varrho(t), t \geq 0, \text { with some } \varrho \in L^{\infty}(0, \infty) \\
\text { (iv) } E \in L(X) \text { and } G: X \rightarrow X, G(0)=0 .
\end{array}\right.
$$

To invert the linear part of $(2.1)$ in the space $L_{w}^{\infty}(0, \infty ; X)$ with an appropriate weight $w$, define the following auxiliary problems. 
- Fundamental solution:

$$
\begin{aligned}
& \varepsilon U_{\varepsilon}^{\prime}(t)+A U_{\varepsilon}(t)-E U_{\varepsilon}(t-\tau)=0, t>0, \\
& U_{\varepsilon}(s)=0 \quad \text { for } s \in(-\tau, 0), \quad U_{\varepsilon}(0)=I, \quad \varepsilon \in\left(0, \varepsilon_{0}\right] .
\end{aligned}
$$

- Homogeneous problem:

$$
\begin{aligned}
& \varepsilon v_{\varepsilon}^{\prime}(t)+A v_{\varepsilon}(t)= \begin{cases}g(t), & t \in(0, \tau), \\
E\left(v_{\varepsilon}(t-\tau)\right), & t>\tau,\end{cases} \\
& v_{\varepsilon}(0)=y, \quad \varepsilon \in\left(0, \varepsilon_{0}\right],
\end{aligned}
$$

where $g:(-\tau, 0) \rightarrow X$ and $y \in X$ are given.

- Inhomogeneous problem:

$$
\begin{gathered}
\varepsilon z_{\varepsilon}^{\prime}(t)+A z_{\varepsilon}(t)-E z_{\varepsilon}(t-\tau)=h(t), \quad t>0, \\
z_{\varepsilon}(s)=0, \quad s \in(-\tau, 0], \quad \varepsilon \in\left(0, \varepsilon_{0}\right],
\end{gathered}
$$

where $h:(0, \infty) \rightarrow X$. Note that $U_{\varepsilon}$ is an operator valued function.

Since $-A$ generates a $C_{0}$-semigroup in $X$, it is clear that for any $\varepsilon \in$ $\left(0, \varepsilon_{0}\right]$ there exists a unique generalized solution $U_{\varepsilon} \in C\left([0, \infty) ; L_{s}(X)\right)$ of (2.4), that is, $U_{\varepsilon}$ satisfies $(2.4)_{2}$ and the integral equation

$$
U_{\varepsilon}(t)=T(t / \varepsilon)+\varepsilon^{-1} \int_{0}^{t} T((t-s) / \varepsilon) E U_{\varepsilon}(s-\tau) d s, \quad t \geq 0 .
$$

Also, there exists $\varrho_{\varepsilon} \in L_{\mathrm{loc}}^{\infty}([0, \infty))$ such that

$$
\left|U_{\varepsilon}(t)\right| \leq \varrho_{\varepsilon}(t) \quad \text { for } t \geq 0 \text { and } \varepsilon \in\left(0, \varepsilon_{0}\right] .
$$

It is a standard result (see e.g. [1]) that the solutions $v_{\varepsilon}$ and $z_{\varepsilon}$ may be expressed in terms of $U_{\varepsilon}$ and $(y, g)$, and of $U_{\varepsilon}$ and $h$, respectively. This is the content of the following two propositions.

2.1. Proposition. Let $y \in X$ and $g \in L^{1}((0, \tau) ; X)$. Then problem (2.5) has a family of generalized solutions $v_{\varepsilon} \in C([0, \infty) ; X), \varepsilon \in\left(0, \varepsilon_{0}\right]$, in the sense that

$$
v_{\varepsilon}(t)= \begin{cases}U_{\varepsilon}(t) y+\varepsilon^{-1} \int_{0}^{t} U_{\varepsilon}(t-s) g(s) d s & \text { for } t \in(0, \tau), \\ U_{\varepsilon}(t) y+\varepsilon^{-1} \int_{0}^{\tau} U_{\varepsilon}(t-s) g(s) d s & \text { for } t>\tau .\end{cases}
$$

If , in addition, $y \in D(A), g \in L^{1}((0, \tau) ; D(A))$ and $E D(A) \subset D(A)$, then the equation in $(2.4)$ is satisfied pointwise a.e. in $(0, \infty)$.

2.2. Proposition. Let $h \in L_{\text {loc }}^{1}([0, \infty) ; X)$. Then problem (2.6) has a family of generalized solutions $z_{\varepsilon} \in C([0, \infty) ; X), \varepsilon \in\left(0, \varepsilon_{0}\right]$, in the sense 
that

$$
z_{\varepsilon}(t)=\varepsilon^{-1} \int_{0}^{t} U_{\varepsilon}(t-s) h(s) d s, \quad t \geq 0 .
$$

If , in addition, $h \in L_{\text {loc }}^{1}([0, \infty) ; D(A))$ and $E D(A) \subset D(A)$, then the equation in (2.6) is satisfied pointwise a.e. in $(0, \infty)$.

Define operators $V_{\varepsilon}$ and $Z_{\varepsilon}$ by

$$
\begin{gathered}
V_{\varepsilon}(y, g)(t)=v_{\varepsilon}(t), \quad t \in[0, \infty), y \in X, g \in L^{1}((0, \tau) ; X), \\
v_{\varepsilon} \text { satisfies }(2.9), \\
Z_{\varepsilon}(h)(t)=z_{\varepsilon}(t), \quad t \in[0, \infty), h \in L_{\mathrm{loc}}^{1}([0, \infty) ; X), \\
z_{\varepsilon} \text { satisfies }(2.10) .
\end{gathered}
$$

In accordance with the definitions of generalized solutions to Problems (2.5), (2.6) it is consistent to define a generalized solution to (2.1) as follows:

2.3. Definition. A function $u_{\varepsilon} \in L_{\mathrm{loc}}^{\infty}([0, \infty) ; X)\left(\varepsilon \in\left(0, \varepsilon_{0}\right]\right)$ is called a generalized solution to problem $(2.1)$ if $u_{\varepsilon}(s)=x(s)$ for $s \in(-\tau, 0]$ and the following integral equation is satisfied:

$$
\begin{aligned}
& \text { (2.12) } \begin{array}{l}
u_{\varepsilon}(t) \\
+\varepsilon^{-1} \int_{0}^{t} U_{\varepsilon}(t-s)\left(E x(s-\tau)+G x(s-\tau)+F u_{\varepsilon}(s)\right) d s, \quad t \in(0, \tau], \\
U_{\varepsilon}(t) x(0)+\varepsilon^{-1} \int_{0}^{\tau} U_{\varepsilon}(t-s)(E x(s-\tau)+G x(s-\tau)) d s \\
+\varepsilon^{-1} \int_{0}^{t} U_{\varepsilon}(t-s)\left(F u_{\varepsilon}(s)+E u_{\varepsilon}(s-\tau)+G u_{\varepsilon}(s-\tau)\right) d s, \quad t>\tau,
\end{array}
\end{aligned}
$$

where $U_{\varepsilon}$ is given by (2.7). Taking into account definitions (2.11), setting $g(t)=E x(t-\tau)+G x(t-\tau)$ for $t \in(0, \tau)$ and $g(t)=0$ for $t>\tau$,

$$
(\bar{G} u)(t)= \begin{cases}0 & \text { for } t \in(0, \tau), \\ E u(t-\tau)+G u(t-\tau) & \text { for } t>\tau\end{cases}
$$

with $u: \mathbb{R}^{+} \rightarrow X$, we can write (2.12) in the form

$$
u_{\varepsilon}(t)=V_{\varepsilon}(x(0), g)(t)+Z_{\varepsilon}\left(F u_{\varepsilon}(\cdot)+\bar{G} u_{\varepsilon}(\cdot)\right)(t), \quad t \geq 0 .
$$

Again, it may be shown in a standard way that the following assertion holds true.

2.4. Proposition. If $x(0) \in D(A), E x(\cdot)+G x(\cdot) \in L^{1}((-\tau, 0) ; D(A))$, $E D(A) \subset D(A)$ and $(2.13)$ has a solution $u_{\varepsilon} \in W^{1,1}(0, T ; X) \cap L^{1}(0 . T ; D(A))$ 
for some $T>0$ and $\varepsilon \in\left(0, \varepsilon_{0}\right]$ then the first equation in (2.1) is satisfied pointwise a.e. in $(0, T)$.

3. Fundamental solution. We start with an investigation of the fundamental solution $U_{\varepsilon}(t)$ of $(2.4)$.

3.1. Lemma. Let assumption (2.3) be satisfied and let

$$
\varrho(t)=M e^{-\alpha t}, \quad t \geq 0, \text { with some constants } M>0, \alpha>|E| .
$$

Assume further that $E$ commutes with $(\lambda I+A)^{-1}$ for some $\lambda$ with $\operatorname{Re} \lambda>-\alpha$. Then for any $\varepsilon>0$ there exists a generalized solution $U_{\varepsilon} \in$ $L_{\mathrm{loc}}^{\infty}((-\tau, \infty) ; L(X))$ of $(2.4)$, and it satisfies

$$
\begin{aligned}
& \left|U_{\varepsilon}(t)\right| \leq M\left(1-\frac{|E| e^{\beta \tau}}{\alpha-\varepsilon \beta}\right)^{-1} e^{-\beta t}, \\
& \varepsilon^{-1} \int_{0}^{\infty} e^{\beta t}\left|U_{\varepsilon}(t)\right| d t \leq M \alpha(\alpha-\varepsilon \beta)^{-1}\left(\alpha-e^{\beta \tau}|E|\right)^{-1}, \\
& \quad \text { for all } t \geq 0, \varepsilon \in\left(0, \varepsilon_{0}\right], \beta \in\left[0, \beta_{0}(\varepsilon)\right) \supset\left[0, \beta_{0}\right),
\end{aligned}
$$

where $\beta_{0}(\varepsilon):=\sup \left\{\beta \in(0, \infty): e^{\beta \tau}|E|<\alpha-\varepsilon \beta\right\}, \beta_{0}:=\beta_{0}\left(\varepsilon_{0}\right)>0$, $\beta_{0}(0+)=\tau^{-1} \log (\alpha /|E|)$.

P r o of. A formal application of the Fourier transform to the function $U_{\varepsilon}$ (extended by zero for $t \leq-\tau)$ suggests that we consider a solution of $(2.4)$ in the form

$$
U_{\varepsilon}(t)= \begin{cases}0, & t<0, \\ \sum_{n=0}^{[t / \tau]} \frac{(t-n \tau)^{n}}{\varepsilon^{n} n !} T\left(\frac{t-n \tau}{\varepsilon}\right) E^{n}, & t \geq 0, \varepsilon>0,\end{cases}
$$

where $[s]$ stands for the integral part of $s$. Let $R(\lambda)=(\lambda I+A)^{-1}$. Then $R(\lambda) \in L(X)$ and, for each $\mu$ with $\operatorname{Re} \mu>-\alpha, R(\mu)=f_{\mu}(R(\lambda))$ with a suitable analytic function $f_{\mu}$. By the functional calculus for bounded linear operators, $E R(\mu)=E f_{\mu}(R(\lambda))=f_{\mu}(R(\lambda)) E=R(\mu) E$. To show that $E$ commutes with $T(s)$ for each $s \geq 0$ we use the Yosida approximation

$$
A_{n}=n^{2} R(-\alpha+n)-(\alpha+n) I, \quad n=1,2, \ldots ;
$$

then $T(s) x=\lim _{n \rightarrow \infty} \exp \left(-s A_{n}\right) x$ for all $x \in X$ and all $s \geq 0$ (see [5, Section 1.3]) and the commutativity follows. It can then be routinely verified that the function $U_{\varepsilon}$ given by (3.3) is a generalized solution of (2.4). We are going to use formula (3.3) to derive the estimates (3.2). Let $\beta \in\left[0, \beta_{0}(\varepsilon)\right.$ ). Setting

$$
v_{\varepsilon}(t)=e^{\beta t} U_{\varepsilon}(t), \quad t \geq 0, \varepsilon \in\left(0, \varepsilon_{0}\right]
$$


$U_{\varepsilon}$ is a generalized solution of (2.4) if and only if $v_{\varepsilon}$ satisfies

$$
\begin{array}{rlrl}
\varepsilon v_{\varepsilon}^{\prime}(t)+(A-\varepsilon \beta I) v_{\varepsilon}(t) & =e^{\beta \tau} E v_{\varepsilon}(t-\tau), & & t \geq 0, \\
v_{\varepsilon}(0) & =I, & & \\
v_{\varepsilon}(s) & =0, & s \in(-\tau, 0) .
\end{array}
$$

A consideration analogous to that for $U_{\varepsilon}$ above leads to the formula

$$
v_{\varepsilon}(t)= \begin{cases}0, & t<0, \\ \sum_{n=0}^{[t / \tau]} \frac{(t-n \tau)^{n}}{\varepsilon^{n} n !} e^{\beta(t-n \tau)} T\left(\frac{t-n \tau}{\varepsilon}\right) e^{n \beta \tau} E^{n}, & t \geq 0, \varepsilon>0 .\end{cases}
$$

We estimate the $n$th term of the sum in (3.6):

$$
\begin{aligned}
a_{n}(t) & :=\left|\frac{(t-n \tau)^{n}}{\varepsilon^{n} n !} e^{\beta(t-n \tau)} T\left(\frac{t-n \tau}{\varepsilon}\right) e^{n \beta \tau} E^{n}\right| \\
& \leq M \frac{(t-n \tau)^{n}}{\varepsilon^{n} n !} \exp \left[\frac{\varepsilon \beta-\alpha}{\varepsilon}(t-n \tau)\right] e^{n \beta \tau}|E|^{n} .
\end{aligned}
$$

Taking logarithm of $a_{n}(t)$ and using the estimate

$$
\log (n !)=\sum_{k=2}^{n} \log k \geq \int_{1}^{n} \log \nu d \nu=n \log n-n,
$$

we obtain

$$
\begin{aligned}
\log a_{n}(t) \leq & \log M+n \log \left(\frac{|E|}{\alpha-\varepsilon \beta}\right) \\
& +\log \left[\sup _{s \geq 0}\left\{s^{n} e^{-s}\right\}\right]+n \beta \tau-(n \log n-n) \\
= & \log M+n\left[\beta \tau+\log \left(\frac{|E|}{\alpha-\varepsilon \beta}\right)\right] .
\end{aligned}
$$

Hence we get

$$
a_{n}(t) \leq M e^{-\kappa n},
$$

where $\kappa:=-\beta \tau-\log (|E| /(\alpha-\varepsilon \beta))$, which is positive by assumption. Consequently, by (3.6)-(3.8) we have

$$
\begin{aligned}
\left|v_{\varepsilon}(t)\right| & \leq \sum_{n=0}^{[t / \tau]} a_{n}(t) \leq M \sum_{n=0}^{[t / \tau]} e^{-\kappa n} \\
& \leq M \sum_{n=0}^{\infty} e^{-\kappa n}=\frac{M}{1-e^{-\kappa}}=M\left(1-\frac{|E| e^{\beta \tau}}{\alpha-\varepsilon \beta}\right)^{-1},
\end{aligned}
$$

and (3.4) yields the first inequality in (3.2). 
Now we prove the second inequality in (3.2). Setting

$$
s=t / \varepsilon, \quad \sigma=\tau / \varepsilon, \quad v(s)=e^{\beta t} U_{\varepsilon}(t),
$$

we obtain

$$
\begin{aligned}
v^{\prime}(s)+(A-\varepsilon \beta I) v(s) & =e^{\beta \tau} E v(s-\sigma), & & s \geq 0, \\
v(0) & =I, & & \\
v(s) & =0, & & s \in(-\sigma, 0) .
\end{aligned}
$$

A similar reasoning to the above leads to the formula

$$
v(s)= \begin{cases}0, & s<0, \\ \sum_{n=0}^{[s / \sigma]} \frac{(s-n \sigma)^{n}}{n !} e^{\varepsilon \beta(s-n \sigma)} e^{n \beta \tau} T(s-n \sigma) E^{n}, & s \geq 0 .\end{cases}
$$

Then we have

$$
\begin{aligned}
J(\varepsilon) & :=\varepsilon^{-1} \int_{0}^{\infty} e^{\beta t}\left|U_{\varepsilon}(t)\right| d t=\int_{0}^{\infty}|v(s)| d s \\
& \leq \sum_{m=0}^{\infty} \int_{m \sigma}^{(m+1) \sigma}\left|\sum_{n=0}^{m} \frac{(s-n \sigma)^{n}}{n !} e^{\varepsilon \beta(s-n \sigma)} e^{n \beta \tau} T(s-n \sigma) E^{n}\right| d s \\
& \leq M \sum_{m=0}^{\infty} \sum_{n=0}^{m} \frac{e^{n \beta \tau}|E|^{n}}{n !} \int_{m \sigma}^{(m+1) \sigma}(s-n \sigma)^{n} e^{-(\alpha-\varepsilon \beta)(s-n \sigma)} d s \\
& =M \sum_{m=0}^{\infty} \sum_{n=0}^{m} \frac{e^{n \beta \tau}|E|^{n}}{n !} \int_{(m-n) \sigma}^{(m+1-n) \sigma} s^{n} e^{-(\alpha-\varepsilon \beta) s} d s .
\end{aligned}
$$

Since

$$
\int s^{n} e^{-\delta s} d s=-\frac{1}{\delta} e^{-\delta s} \sum_{l=0}^{n} \frac{n ! s^{n-l}}{(n-l) ! \delta^{l}},
$$

we find that

$$
\begin{aligned}
J(\varepsilon) \leq & -\frac{M}{\alpha-\varepsilon \beta} \sum_{m=0}^{\infty} \sum_{n=0}^{m} e^{n \beta \tau}|E|^{n} \\
& \times \sum_{l=0}^{n} \frac{1}{(\alpha-\varepsilon \beta)^{l}(n-l) !}\left[s^{n-l} e^{-(\alpha-\varepsilon \beta) s}\right]_{s=(m-n) \sigma}^{(m+1-n) \sigma} \\
\leq & \frac{M}{\alpha-\varepsilon \beta} \sum_{m=0}^{\infty} \sum_{n=0}^{m} e^{n \beta \tau}|E|^{n} \sum_{l=0}^{n} \frac{\sigma^{n-l}}{(\alpha-\varepsilon \beta)^{l}(n-l) !} \\
& \times\left[(m-n)^{n-l} e^{-(\alpha-\varepsilon \beta)(m-n) \sigma}-(m+1-n)^{n-l} e^{-(\alpha-\varepsilon \beta)(m+1-n) \sigma}\right]
\end{aligned}
$$




$$
\begin{aligned}
= & \frac{M}{\alpha-\varepsilon \beta} \sum_{m=0}^{\infty} \sum_{n=0}^{m} \sum_{l=0}^{n} \frac{e^{n \beta \tau}|E|^{n} \sigma^{n-l}}{(\alpha-\varepsilon \beta)^{l}(n-l) !}(m-n)^{n-l} e^{-(\alpha-\varepsilon \beta)(m-n) \sigma} \\
& -\frac{M}{\alpha-\varepsilon \beta} \sum_{m=1}^{\infty} \sum_{n=0}^{m-1} \sum_{l=0}^{n} \frac{e^{n \beta \tau}|E|^{n} \sigma^{n-l}}{(\alpha-\varepsilon \beta)^{l}(n-l) !}(m-n)^{n-l} e^{-(\alpha-\varepsilon \beta)(m-n) \sigma} \\
= & \frac{M}{\alpha-\varepsilon \beta}+\frac{M}{\alpha-\varepsilon \beta} \sum_{m=1}^{\infty} \sum_{l=0}^{m} \frac{e^{m \beta \tau}|E|^{m} \sigma^{m-l}}{\alpha^{m}(m-l) !}(m-m)^{m-l} e^{-(\alpha-\varepsilon \beta)(m-m) \sigma} \\
= & \frac{M}{\alpha-\varepsilon \beta}+\frac{M}{\alpha-\varepsilon \beta} \sum_{m=1}^{\infty} \frac{e^{m \beta \tau}|E|^{m}}{\alpha^{m}}=M \alpha(\alpha-\varepsilon \beta)^{-1}\left(\alpha-e^{\beta \tau}|E|\right)^{-1},
\end{aligned}
$$

and the second inequality in (3.2) follows immediately.

4. Uniform stability. In this last section we present a uniform stability theorem for problem (2.1).

4.1. TheOREM. Let the assumptions of Lemma 3.1 hold, together with the following additional condition:

(v) there exists $r_{0}>0$ and a continuous nondecreasing function $\lambda$ : $\left[0, r_{0}\right) \rightarrow \mathbb{R}^{+}$with $\lambda(0)=0$ such that for any $r \in\left(0, r_{0}\right)$ we have

$$
\max \{|F(u)-F(v)|,|G(u)-G(v)|\} \leq \lambda(r)|u-v| \quad \text { for } u, v \in B_{r}(0 ; X) .
$$

Then there exists $R>0$ such that if

$$
\|x\|_{L^{\infty}(-\tau, 0)}+|x(0)| \leq R
$$

then the corresponding generalized solution $u_{\varepsilon}(t)$ of $(2.1)$ exists and satisfies (4.2) $\left|u_{\varepsilon}(t)\right| \leq C(\beta)\left(\|x\|_{L^{\infty}(-\tau, 0)}+|x(0)|\right) e^{-\beta t} \quad$ for $t \geq 0$ and $\varepsilon \in\left(0, \varepsilon_{0}\right]$, with a constant $C(\beta)$ independent of the function $x$, and $\beta$ in the same range as in Lemma 3.1.

Proof. Let $\beta \in\left(0, \beta_{0}(\varepsilon)\right)$, where $\beta_{0}(\varepsilon)$ is defined as in Lemma 3.1, $\varepsilon \in\left(0, \varepsilon_{0}\right]$. Define $w(t)=e^{\beta t}$ for $t \geq 0$, and let

$$
\begin{aligned}
H_{\varepsilon}(u)(t):= & U_{\varepsilon}(t) x(0)+\varepsilon^{-1} \int_{0}^{t} U_{\varepsilon}(t-s) g(s) d s \\
& +\varepsilon^{-1} \int_{0}^{t} U_{\varepsilon}(t-s)[F u(s)+\bar{G} u(s)] d s
\end{aligned}
$$

for $u \in L_{w}^{\infty}(0, \infty ; X), t \geq 0$ with $x \in L^{\infty}(0, \tau), x(0) \in X$ given, and $g$ and $\bar{G}$ as in (2.13), (2.14). By Definition 2.3 and (2.14) it is sufficient to prove that if (4.1) is satisfied with $R>0$ small enough, then for each $\varepsilon \in\left(0, \varepsilon_{0}\right]$ the mapping $H_{\varepsilon}$ has a fixed point in $L_{w}^{\infty}(0, \infty ; X)$. As in the proof of Theorem 3.3 of [3] we make use of the Banach contraction principle 
in a sufficiently small ball $B_{r}\left(0 ; L_{w}^{\infty}(0, \infty ; X)\right)$, where $r>0$. Then by $(3.2)$ for $u \in B_{r}\left(0, L_{w}^{\infty}(0, \infty ; X)\right)$ we have

$$
\begin{aligned}
e^{\beta t}\left|H_{\varepsilon}(u)(t)\right| \leq & e^{\beta t}\left|U_{\varepsilon}(t)\right| \cdot|x(0)|+\varepsilon^{-1} \int_{0}^{t} e^{\beta(t-s)}\left|U_{\varepsilon}(t-s)\right| d s\|g\|_{w} \\
& +2 \varepsilon^{-1} \int_{0}^{t} e^{\beta(t-s)}\left|U_{\varepsilon}(t-s)\right| d s \lambda(r)\|u\|_{w} \\
\leq & M\left(1-\frac{|E| e^{\beta \tau}}{\alpha-\varepsilon \beta}\right)^{-1} R \\
& +M \alpha(\alpha-\varepsilon \beta)^{-1}\left(\alpha-e^{\beta \tau}|E|\right)^{-1}(\lambda(R)+|E|)\|x\|_{L^{\infty}(-\tau, 0)} e^{\beta \tau} \\
& +2 \lambda(r) M \alpha(\alpha-\varepsilon \beta)\left(\alpha-e^{\beta \tau}|E|\right)^{-1} r \\
\leq & \operatorname{const} \cdot(R+\lambda(r) r) \leq r,
\end{aligned}
$$

the last inequality holding when $R$ and $r$ are sufficiently small.

Similarly we have

$$
\begin{aligned}
e^{\beta t}\left|H_{\varepsilon}(u)(t)-H_{\varepsilon}(v)(t)\right| & \leq 2 \varepsilon^{-1} \int_{0}^{t} e^{\beta(t-s)}\left|U_{\varepsilon}(t-s)\right| d s \lambda(r)\|u-v\|_{w} \\
& \leq \mathrm{const} \cdot \lambda(r)\|u-v\|_{w}
\end{aligned}
$$

and $r>0$ can be chosen so that const $\lambda(r)<1$. So we have proved that, for sufficiently small numbers $R>0$ and $r>0, H_{\varepsilon}$ maps the ball $B_{r}\left(0 ; L_{w}^{\infty}(0, \infty ; X)\right)$ into itself and is a contraction. The Banach contraction principle implies that, for any $\varepsilon>0$ and $x$ satisfying (4.1), there exists a unique fixed point $u_{\varepsilon}$ of $H_{\varepsilon}$ in $B_{r}\left(0 ; L_{w}^{\infty}(0, \infty ; X)\right)$. This is clearly the generalized solution of (2.1) satisfying (4.2).

4.2. ExAmple. As an example of application let us consider the following problem:

$$
\begin{aligned}
& \varepsilon \frac{\partial u_{\varepsilon}}{\partial t}(x, t)-\sum_{j, k=1}^{N} \frac{\partial}{\partial x_{j}}\left(a_{j k}(x) \frac{\partial u_{\varepsilon}}{\partial x_{k}}(x, t)\right)-b u_{\varepsilon}(x, t) \\
& =f\left(u_{\varepsilon}(x, t)\right)+g\left(u_{\varepsilon}(x, t-\tau)\right), \\
& x \in \Omega \subset \mathbb{R}^{N}, t>0, \varepsilon \in\left(0, \varepsilon_{0}\right]\left(\varepsilon_{0}>0\right), \\
& u_{\varepsilon}(x, t)=0, \quad x \in \partial \Omega, t>0, \\
& u_{\varepsilon}(x, s)=\varphi(x, s), \quad x \in \Omega, s \in(-\tau, 0](\tau>0) .
\end{aligned}
$$

Here $\Omega$ is a bounded domain with $C^{2}$-boundary $\partial \Omega ; a_{j k} \in C^{2}(\bar{\Omega}), a_{j k}=a_{k j}$ for $j, k=1, \ldots, n ; \sum_{j, k=1}^{N} a_{j k} \xi_{j} \xi_{k} \geq c_{0}|\xi|^{2}$ for $\xi \in \mathbb{R}^{N}$ with $c_{0}>0 ; b \in \mathbb{R}$; 
$f, g: \mathbb{R}^{N} \rightarrow \mathbb{R}, f(0)=g(0)=0 ; \varphi: \Omega \times(-\tau, 0] \rightarrow \mathbb{R}$. Moreover, assume that

$\left(\mathrm{v}^{\prime}\right) f, f^{\prime}, g, g^{\prime}$ are locally Lipschitz continuous and there exists $r_{0}>0$ and a continuous function $\lambda=\lambda(r), r \in\left[0, r_{0}\right), \lambda(0)=0$ such that for any $r \in\left(0, r_{0}\right]$ we have $\max \left\{|f(u)-f(v)|,\left|f^{\prime}(u)-f^{\prime}(v)\right|,|g(u)-g(v)|, \mid g^{\prime}(u)-\right.$ $\left.g^{\prime}(v) \mid\right\} \leq \lambda(r)|u-v|$ for $u, v \in \mathbb{R}$ satisfying $\max \{|u|,|v|\} \leq r$.

Let $p>N$ and $X=\stackrel{\circ}{W}^{1, p}(\Omega)$. It is a standard result [5] that the operator $-A$ defined by $A v=\sum_{j, k=1}^{N} \frac{\partial}{\partial x_{j}}\left(a_{j k}(x) \frac{\partial v}{\partial x_{k}}\right)$ for $v \in W^{2, p}(\Omega) \cap \stackrel{\circ}{W}^{1, p}(\Omega)$ generates an exponentially decreasing semigroup on $L^{p}(\Omega)$. This semigroup is invariant on $X$ and is also exponentially decreasing (see e.g. [3], Proposition 6.1), which means that the assumptions (i) and (iii) of (2.3) are satisfied, and it can easily be shown (see [3], proof of Proposition 6.1) that $\alpha$ in (3.1) can be chosen as

$$
\alpha:=4 c_{0} m \frac{p-1}{p^{2}},
$$

where $m=\inf \left\{\int_{\Omega}|\nabla v|^{2} d x / \int_{\Omega} v^{2} d x: v \in \stackrel{\circ}{W}^{1,2}(\Omega), v \neq 0\right\}$. Assuming $b<\alpha$ we meet the demands of (3.1). Finally, it is a routine matter to verify from $\left(\mathrm{v}^{\prime}\right)$ the assumption (ii) and (iv) of (2.3) and the assumption (v) of Theorem 4.1, since $X \hookrightarrow L^{\infty}(\Omega)$. Then Theorem 4.1 has the following consequence:

COROLlaRY 4.3. Under the above assumptions there exists $R>0$ such that if $\|\varphi(\cdot, \cdot)\|_{L^{\infty}\left(-\tau, 0 ; W^{1, p}(\Omega)\right)}+\|\varphi(\cdot, 0)\|_{W^{1, p}(\Omega)} \leq R$ then the corresponding generalized solution of the problem (4.4) exists with values in $\stackrel{\circ}{W}^{1, p}(\Omega)$ and satisfies

$$
\begin{array}{r}
\left\|u_{\varepsilon}(\cdot, t)\right\|_{W^{1, p}(\Omega)} \leq C(\beta)\left(\|\varphi(\cdot, \cdot)\|_{L^{\infty}\left(-\tau, 0 ; W^{1, p}(\Omega)\right)}+\|\varphi(\cdot, 0)\|_{W^{1, p}(\Omega)}\right) e^{-\beta t} \\
\text { for } t \geq 0 \text { and } \varepsilon \in\left(0, \varepsilon_{0}\right],
\end{array}
$$

with a constant $C(\beta)$ independent of the function $u_{0}$ and $\beta$ in the same range as in Lemma 3.1, $\alpha$ being given by (4.5).

Let us note that the diffusive functional differential equations of the type (4.4) are important in biological models (cf. [4]).

Acknowledgements. The authors are indebted to the referee for his careful reading of the manuscript and valuable remarks which helped to improve the quality of the paper.

\section{References}

[1] J. K. Hale, Theory of Functional Differential Equations, Springer, New York, 1977. 
[2] J. S. Jung, J. Y. Park and H. J. Kang, Asymptotic behavior of solutions of nonlinear functional differential equations, Internat. J. Math. Math. Sci. 17 (1994), 703-712.

[3] J. J. Koliha and I. Straškraba, Stability in nonlinear evolution problems by means of fixed point theorems, Comment. Math. Univ. Carolin. 38 (1) (1997), to appear.

[4] S. Murakami, Stable equilibrium point of some diffusive functional differential equations, Nonlinear Anal. 25 (1995), 1037-1043.

[5] A. Pazy, Semigroups of Linear Operators and Applications to Partial Differential Equations, Springer, New York, 1983.

[6] C. C. Travis and G. F. Webb, Existence and stability for partial functional differential equations, Trans. Amer. Math. Soc. 200 (1974), 395-418.

[7] - - - Partial differential equations with deviating arguments in the time variable, J. Math. Anal. Appl. 56 (1976), 397-409.

[8] T. Wang, Stability in abstract functional differential equations. Part I. General theorems, ibid. 186 (1994), 534-558.

[9] - Stability in abstract functional differential equations, Part II. Applications, ibid. 186 (1994), 835-861.

[10] G. F. W ebb, Asymptotic stability for abstract nonlinear functional differential equations, Proc. Amer. Math. Soc. 54 (1976), 225-230.

Department of Mathematics

University of Melbourne

Parkville, Victoria 3052

Australia

E-mail: j.koliha@maths.unimelb.edu.au
Mathematical Institute Academy of Sciences of the Czech Republic

11567 Praha 1

Czech Republic E-mail: strask@mbox.cesnet.cz 